\title{
Causal Effects, Migration and Legacy Studies
}

\author{
Moritz Marbach|*
}

August 2022

\begin{abstract}
Political scientists have long been interested in the persistent effects of history on contemporary behavior and attitudes. To estimate legacy effects, studies often compare people living in places that were historically exposed to some event and those that were not. Using principal stratification, we provide a formal framework to analyze how migration limits our ability to learn about the persistent effects of history from observed differences between historically exposed and unexposed places. We state the necessary assumptions about movement behavior to causally identify legacy effects. We highlight that these assumptions are strong; therefore, we recommend that legacy studies circumvent bias by collecting data on people's place of residence at the exposure time. Reexamining a study on the persistent effects of US civil-rights protests, we show that observed attitudinal differences between residents and non-residents of historic protest sites are more likely due to migration rather than attitudinal change.
\end{abstract}

\footnotetext{
${ }^{*}$ University College London, m.marbach@ucl.ac.uk. I am grateful to Achim Ahrens, Joan Barceló, Daniel Bischof, Carlo Horz, Jonathan Homola, Gloria Gennaro, Eric Lewis, Guido Ropers as well as the participants of Texas A\&M's CIPEG Workshop, PolMeth Europe 2022, EPSA 2022, MPSA 2022 for feedback on earlier versions of this paper.
} 


\section{Introduction}

Political scientists have long been interested in the persistent effect of local historical events, policies, or institutional changes on contemporary political behavior and attitudes of individuals. At the heart of these studies is often a comparison of the attitudes and behavior of residents in historically exposed and unexposed places. For example, a recent study by Homola et al. (2020) shows that Germans living closer to former Nazi concentration camps today tend to be more xenophobic, intolerant, and more likely to vote for a far-right party. Another example is Rozenas and Zhukov (2019) who link Stalin's terror by hunger to Ukrainian citizens' loyalty and opposition toward Moscow across eight decades. A third example is Mazumder (2018) demonstrating that respondents living in counties where civil rights protests took place in the 1960s are less likely to harbor racial resentment against Blacks 11

How much can be learned about the causal effect of historical events on contemporary attitudes and behavior from observed differences between residents in historically exposed and unexposed places? To the extent that people can move after the historical event of interest, any comparison between contemporary outcomes of people in places historically exposed with people in places historically unexposed is not a comparison of exposed and unexposed people alone. For example, people living in historically exposed places today includes people that lived in these places at the time of exposure but it also includes people that lived elsewhere and may have not been subject to the exposure. Therefore, observed differences will not necessarily equal the causal effect of the exposure on individuals even if the exposure was completely randomly assigned across places. Authors of legacy studies recognize that migration possess a challenge to the interpretation of their results. However, the problem is that we lack a formal framework to analyze how and when migration matters for our ability to identify the causal effect of historic events on contemporary outcomes.

In this paper, we address this gap in the literature by providing such a framework. We focus on a setting in which the effect of a one-time shock on an individual's own outcomes is of interest $2^{2}$ Similar to the four complier types in instrumental variable estimation Angrist et al., 1996), we define four latent mover types (never-movers, always-movers, escapees, and followers) and show how the presence of these mover types in the population leads to, what we refer to as, post-treatment sorting bias. We consider a setup in which an exposure status is randomly assigned across places and the distribution of latent mover types is balanced

\footnotetext{
${ }^{1}$ For a recent review on legacy studies, see Cirone and Pepinsky (2021).

${ }^{2}$ In the discussion section, we consider the role of sorting in settings in which the exposure is permanent rather than a one-time shock, exposure-outcome effects span generations and outcomes are not measured on the individual level but on the community level.
} 
(see Figure 1). Once the exposure is realized, some types sort from exposed to unexposed places (and vice versa). Sorting, that is movement between exposed and unexposed places, leads to a distribution of types that is unbalanced at the time outcomes are measured which is the source of post-treatment sorting bias.

We demonstrate that even if sorting is not caused by the exposure (there are only neverand always-movers), the causal effect cannot be recovered without bias. The reason is that some units sort regardless of their exposure status (the always-movers) thus diluting the average treatment effect. In the example displayed in Figure 1, exposed always-movers swapped places with unexposed always-movers mixing exposed and unexposed units. We demonstrate that under general conditions (all four types are present), post-treatment sorting bias can be of any magnitude and go in any direction. The intuition is that escapees fleeing from the exposure are concentrating in the unexposed places while followers congregate in exposed places (see again Figure 1). If the potential outcomes from these two types differ ex ante, differences between exposed and unexposed places will be partially due to this heterogeneity.

(A) Past: Assignment of exposure status
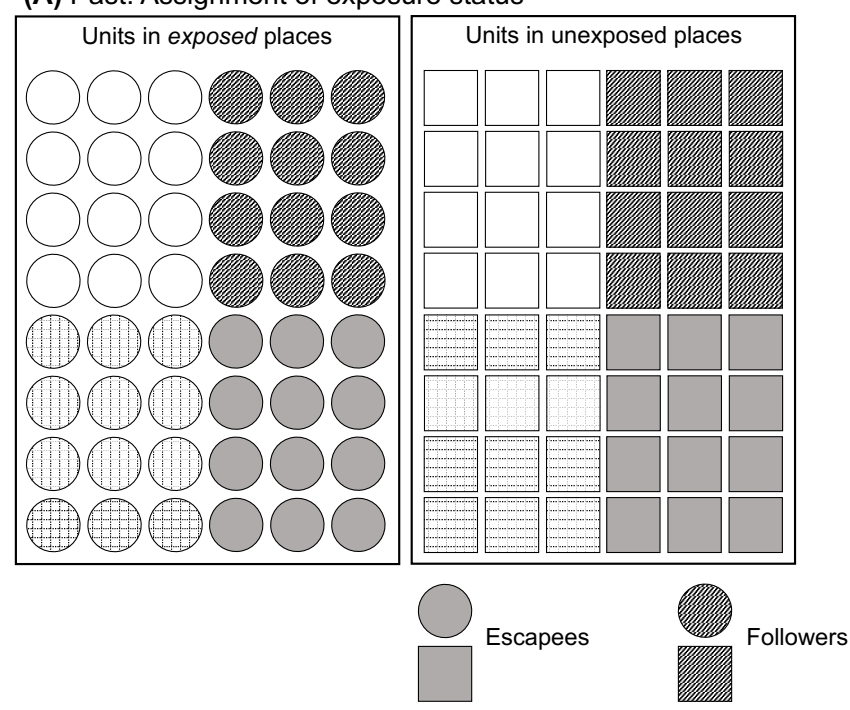

(B) Present: Measurement of outcomes

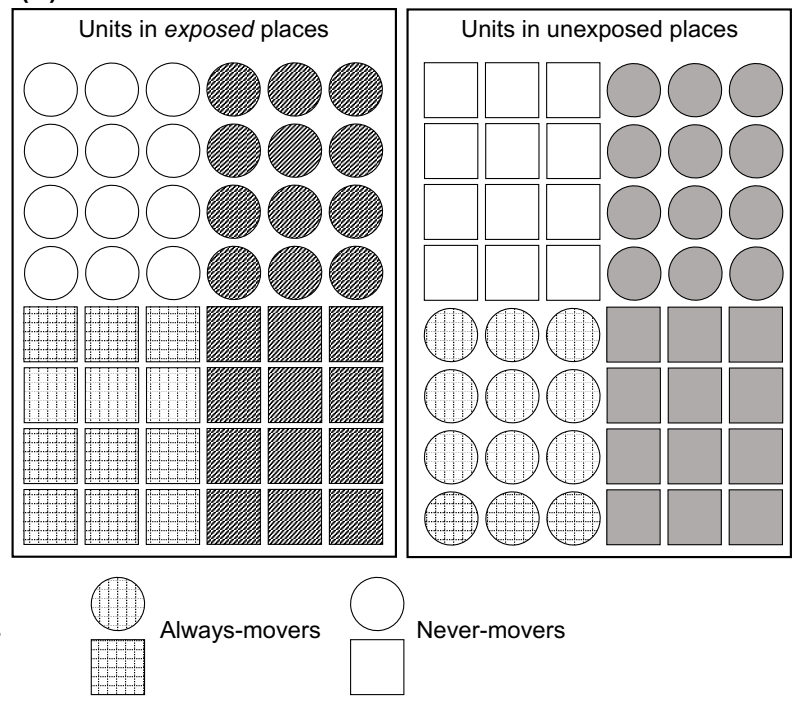

Figure 1: At the time of exposure assignment the distribution of four latent mover types is balanced between exposed and unexposed places (Panel A) but at the time of outcome measurement (after sorting) the distribution is unbalanced (Panel B). The unbalanced type distribution is the reason for why the comparison of units in exposed and unexposed places differs from the comparison of exposed and unexposed units. While the latter is the causal effect of the exposure, the former is distorted by post-treatment sorting bias. As the exposed and unexposed always-movers swap places, their presence dilutes the causal effect while the concentration of escapees and followers in exposed or unexposed places distorts the causal effect if these types are ex ante heterogeneous in their outcomes. 
The solution to post-treatment sorting bias is simple from a theoretical perspective: Studies should compare historically exposed and unexposed units instead of comparing units living in exposed and unexposed places. While theoretically straightforward, the practical implementation requires that studies find data - or collect their own data - that includes information on where someone lived at the time of the exposure. An example for this approach is a study by Barceló (2021) who examines the effect of war exposure on civic engagement. Rather than correlating civic engagement with the historic conflict intensity in respondents' contemporary place of residence, the author obtained data on respondents' place of residence approximately at the time of the war to construct the conflict-exposure variable. If authors are unable to obtain the required data, our paper might help authors to discuss the magnitude and the direction of the sorting bias one may expect.

Throughout this paper we reanalyze a study on the persistent effect of the US civil-rights protests as a running example (Mazumder, 2018). In this important study, Mazumder argues that attitudes of Whites toward Blacks shifted in counties that were scenes of civil rights protests in the 1960s. The empirical analysis relies on contemporary survey responses among White respondents in the cross-sections of 2006-2011 from the Cooperative Congressional Election Study (CCES). More specifically, the analysis uses survey responses and respondents' current place of residence to construct county-level averages measuring political attitudes and regresses those on a binary indicator if any civil rights protests occurred in the same county between 1960-65. Relying on a selection-on-observable assumption, the estimates suggest that survey respondents living in US counties where civil rights protests occurred are more likely to support affirmative action, harbor less political resentment toward Blacks, and are more likely to identify as Democrats.

We use this study as a running example since the publicly available survey data provide additional information on respondents' place of residence when they were 10 and 17 years old. This additional data allows us to directly observe whether some survey respondents were exposed to the protests in the 1960s. Using the historic exposure status in the main regressions eliminates most sorting bias by definition. Leveraging this new data, we find little evidence for a legacy effect of these protests on political resentment toward Blacks, support for affirmative action, or Democratic party identification. The absence of treatment effects suggests that the observed differences between protest and non-protest counties are due to post-treatment sorting bias. Consistent with this statement and the White-Flight hypothesis (Duncan and Duncan, 1957; Taeuber and Taeuber, 1965), we find evidence that protests increased the likelihood of Whites leaving protest counties in the 1960s.

Most legacy studies including this one grapple with sorting. Typically, studies present evidence that demographic measures such as net-migration or total population change are not 
differential between historically exposed and unexposed places and therefore, so the argument goes, can not confound the treatment-outcome effect. Using measures of migration, studies also use mediation analysis to demonstrate that there is a direct effect of the exposure on the outcome. Using the framework, we discuss if these strategies help to isolate the causal effect from the post-treatment sorting bias. We also discuss the role of sorting in other settings, including settings in which the exposure is permanent rather than a one-time shock, exposure-outcome effects span generations and outcomes are measured on the cluster rather than the individual level. The discussion highlights that sorting is not less important in these settings.

The main contribution of this paper is that it conceptualizes and characterizes the consequences of unobserved post-treatment sorting in legacy studies analyzing political behavior and attitudes. We highlight that post-treatment sorting bias can be thought of as an instance of measurement-error bias. Relying on the principal strata framework (Frangakis and Rubin, 2004), we discuss the difficulties of removing sorting bias based on behavioral assumptions about mover types. We detail a series of assumptions assuring that sorting bias attenuates the treatment effect toward zero and therefore mimics the bias due to non-differential measurement error in the exposure variable (Aigner, 1973; Lewbel, 2007). We highlight that the necessary assumptions are stringent and often unrealistic. We, therefore, side with the increasing scepticism toward the convenient assumption of non-differential measurement in realistic settings (e.g., Imai and Yamamoto, 2010). This study also contributes to a growing methodological literature on how to isolate persistent effects from observational data (e.g., Kelly, 2019); see also Voth (2021) for a review. Empirically, we contribute to the growing body of literature that highlights how internal migration shapes (and reshapes) political geography in the United States (e.g., Cantoni and Pons, 2022; Brown and Enos, 2021).

\section{Unobserved Mover Types}

We consider a population of $N$ units. Let $C_{i}$ denote an indicator to which cluster a unit $i$ belongs to and let $Z_{i}$ denote the binary (treatment) exposure $!^{3}$ Throughout this paper, we assume that the treatment is assigned at the cluster level (spatial treatment), such that the units in a cluster $c$ are either all exposed or not exposed, that is, $Z_{c}=\sum_{i: C_{i}=c} Z_{i}$ and $Z_{c} \in 0,1$. If unit $i$ lived in a cluster that was exposed (treatment cluster), the exposure of this unit takes the value 1 . If the unit lived in a cluster that was not exposed (control cluster), the exposure of this unit takes the value 0 . In our running example, a respondent

\footnotetext{
${ }^{3}$ The binary exposure setting is a useful starting point as it lends itself to the simple typology of mover types that we introduce here.
} 
is exposed if they lived in county with civil rights protests at the time when these protest happened.

We focus on a situation in which the exposure is a shock (e.g., protest events) rather than institutional change (e.g., a new local law). The key difference between these two types of exposures is that a shock is a one-time event while an institutional change persists over time. For the latter, the notion of exposed and unexposed is more complicated as units differ (possibly endogenously) in how long they are affected by the exposure. We return to this point in the discussion section below.

After the exposure is realized but before outcomes are measured, units may move from treatment to control clusters (and vice versa) [4 Since units' choice to move may depend on exposure, we define two potential movement choices for each unit; one for when the unit is exposed, $M_{i}(1)$, and one when it is unexposed, $M_{i}(0)$. Depending on a unit's exposure status, only one of the two movement choices is realized and, at least in principle, could be observed. The realized movement, $M_{i}$, measures if a unit decided to move $\left(M_{i}=1\right)$ or not $\left(M_{i}=0\right)$. In our setup, it is sufficient to consider moves from a treatment to a control cluster or from a control cluster to a treatment cluster (but ignore moves across treatment clusters or across control clusters).

Similarly to the compliance types defined by (Angrist et al., 1996), we define four different mover types depending on units' potential movement choices (see table 1). Units that always move regardless of exposure status are referred to as always-movers while units that never move are characterized as never-movers. When a unit only moves when exposed, we refer to such a unit as escapee. Similarly, when a unit only moves when not exposed, we refer to such a unit as follower. For the last two types, the exposure prompts a unit to move: Escapees flee from treatment clusters while followers move toward treatment clusters. Let $S_{i}$ be an variable encoding a unit's moving type: never-mover $(N)$, always-mover $(A)$, escapee $(E)$, or follower $(F)$.

\begin{tabular}{ll}
\hline Types & Exposure and (potential) movement \\
\hline Always-mover & $M(0)=1 M(1)=1$ \\
Never-mover & $M(0)=0 M(1)=0$ \\
Escapee & $M(0)=0 M(1)=1$ \\
Follower & $M(0)=1 M(1)=0$ \\
\hline
\end{tabular}

Table 1: Potential movement and the exposure defining four subpopulations.

\footnotetext{
${ }^{4}$ We abstract from the possibility that units may die or move to a cluster that is neither treatment nor control (i.e., that there is attrition). In the civil-rights protest study, that means, for example, that there is no emigration to other countries.
} 
Exposure and units' mover type jointly determine a unit's residency at the time when outcomes are measured. We define a unit's residency $R_{i}$, by measuring if a unit resides in a cluster (historically) exposed to the treatment $\left(R_{i}=1\right)$ or not $\left(R_{i}=0\right)$ at the time when outcomes are measured. More specifically, we can write $R_{i}$ as a function of potential movement and exposure, that is, $R_{i}\left(Z_{i}\right)=Z_{i} \cdot\left(1-M_{i}\left(Z_{i}\right)\right)+\left(1-Z_{i}\right) \cdot M_{i}\left(Z_{i}\right)$, or in terms of realized movement and exposure, that is, $R_{i}=Z_{i} \cdot\left(1-M_{i}\right)+\left(1-Z_{i}\right) \cdot M_{i}$. In the civil-rights protest study, $R_{i}$ measures if respondents currently lives in a county that was the site of protests in the 1960s (or not).

Let $Y_{i}(r, z)$ be the potential outcome for a unit $i$ with exposure $z$ and residency $r$. We define four instead of two potential outcomes to accommodate situations in which moving affects the outcome. Let $Y_{i}$ be the realized outcome. For each unit, only one potential outcome is realized. Without loss of generality, we leave the time difference between exposure and outcome measurement implicit! 5

We assume that one seeks to identify the average treatment effect (ATE) of $Z$ which is defined as $\delta=\mathbb{E}[Y(R(1), 1)-Y(R(0), 0)]$. We say that $\delta$ is a legacy effect as it is measured some time after the exposure occurred. Throughout this paper, and to facilitate the intuition in how (unobserved) sorting complicates causal inference, we abstract from any confounding and assume that the exposure is randomly assigned:

\section{Assumption 1 Exposure randomization}

$Y(0,0), Y(1,1), Y(1,0), Y(0,1) \Perp Z$.

In our running example, the author does not assume that protests are randomly assigned but rather that after conditioning on a series of observables, it is as-if random if someone got exposed (or not) to these protests. To facilitate the intuition, we discuss sorting bias in the context of a completely randomized exposure rather than a conditional independent exposure.

The focus of this paper is on the identifiability of units' average treatment effect. Not all legacy studies target this estimand. In cluster-level legacy studies, the target estimand is the difference between a cluster's potential outcome when exposed and a cluster's potential outcome when not exposed. We discuss the role of sorting in these cluster-level legacy studies in the discussion section.

\footnotetext{
${ }^{5}$ The definition of the potential outcomes implies that there is no interference between units. In the setting considered here, the stable unit treatment assumption rules out, for example, that the potential outcomes of an unexposed never-mover $j$ are dependent on the arrival of an exposed escapee $i$ in the same cluster. In the running example, this assumption could be violated if, for example, White escapees affect the racial resentment of Whites at their destination.
} 


\section{Sorting Bias in Regressions}

We assume that units sort if there are at least some units for which $R_{i} \neq Z_{i}$, that is, if at least some units move from any treatment cluster to any control cluster (or vice versa) after the exposure was realized. When units move from a treatment cluster to a control cluster (and vice versa) before the exposure is realized, we say that units select rather than sort. The focus of this study is on post-treatment sorting rather than pre-treatment self-selection.

With (cluster-level) randomization and information about units' exposure status at the time of exposure, the average treatment effect (ATE) is identified. For example, the coef-

ficient $\delta$ estimates the ATE when regressing the observed outcomes $\left(Y_{i}^{\text {obs }}\right)$ on the exposure indicator $\left(Z_{i}\right)$ :

$$
Y_{i}=\alpha+\delta Z_{i}+\epsilon_{i}
$$

However, as explained above, we assume that information about $Z_{i}$ is not available and therefore, we compare treatment and control clusters instead(as defined by $R_{i}$ ):

$$
Y_{i}=\alpha^{\prime}+\delta^{\prime} R_{i}+\epsilon_{i}^{\prime}
$$

In this regression, the coefficient $\delta^{\prime}$ does not generally estimate the ATE. The estimates from such a regression are presented in the study on the persistent effect of the civil-rights protests. The author regresses measure of political resentments on an indicator of whether or not a respondent lived at the time of a survey in a county with civil-rights protests in the 1960s.

Post-treatment sorting bias is the difference $\delta^{\prime}-\delta$. Conceptually, sorting bias originates in the imperfect observability of treatment exposure. Since a unit's residency $\left(R_{i}\right)$ is a function of a unit's exposure $\left(Z_{i}\right)$, we can think of $R_{i}$ as a contaminated version of $Z_{i}$ and rewrite the regression equation 2 as a function of realized movement and exposure:

$$
\begin{aligned}
Y_{i} & =\alpha^{\prime}+\delta^{\prime}\left(Z_{i}\left(1-M_{i}+\left(1-Z_{i}\right) M_{i}\right)+\epsilon_{i}^{\prime}\right. \\
& =\alpha^{\prime}+\delta^{\prime} Z_{i}+\delta^{\prime} M_{i}-2 \delta^{\prime} Z_{i} M_{i}+\epsilon_{i}^{\prime}
\end{aligned}
$$

This regression equation highlights that regressing the observed outcome on the residency indicator is identical to regressing the observed outcome on i) the treatment exposure indi- 
cator, ii) the realized movement indicator and iii) the (scaled) interaction between the two while simultaneously constraining the coefficients for these three terms to the same value. While this equation does not reveal much about the direction or magnitude of the bias, it highlights that $\delta^{\prime}$ is unlikely to equal the ATE (here $\delta$ ) in general.

\section{Anatomy of Sorting Bias}

We next decompose the $\delta^{\prime}$ into a series of contrasts for the different mover types defined above. Different from the regressions in the previous section, we make no constant-effect assumption and use the mover typology introduce earlier. To facilitate the intuition of this decomposition, consider table 2 which stratifies the population by realized residency $(R)$ and exposure $(Z)$. Each cell consists of units from two different types. Based on $Z_{i}$ and $R_{i}$ alone, these two different types per cell cannot be distinguished. For example, units in the upper left cell could be escapees (units that only move when exposed) or never-movers (units that never move no matter what the exposure). In the upper right cell, units could be escapees (they left for a control cluster after getting exposed) or always-movers (units that always move no matter the exposure). The same logic applies to the cells in the lower row.

\begin{tabular}{cccc}
\hline \multicolumn{5}{c}{0} \\
& & 1 \\
\hline \multirow{2}{*}{$R$} & 0 & escapee \& never-mover & escapee \& always-mover \\
& 1 & follower \& always-mover & follower \& never-mover \\
\hline
\end{tabular}

Table 2: Stratification of the population by exposure $(Z)$ and realized residency status $(R)$ into never-movers, always-mover, escapee and follower.

Maintaining the randomized-exposure assumption (A1), we can decompose the observed difference between treatment and control clusters, $\delta^{\prime}$, (the difference between outcomes from units in the lower row and the outcomes from units in the upper row) as follows:

$$
\begin{aligned}
\delta^{\prime}= & \mathbb{E}[Y \mid R=1]-\mathbb{E}[Y \mid R=0] \\
= & (p(S=N \mid R=1) \mathbb{E}[Y(1,1) \mid R=1, S=N]-p(S=N \mid R=0) \mathbb{E}[Y(0,0) \mid R=0, S=N])+ \\
& (p(S=A \mid R=1) \mathbb{E}[Y(1,0) \mid R=1, S=A]-p(S=A \mid R=0) \mathbb{E}[Y(0,1) \mid R=0, S=A])+ \\
& (p(S=F \mid R=1) \mathbb{E}[Y \mid R=1, S=F]-p(S=E \mid R=0) \mathbb{E}[Y \mid R=0, S=E]))
\end{aligned}
$$

This decomposition shows that the observed difference is the sum of three contrasts. The first contrast is the difference between exposed units and unexposed units among never- 
movers. In terms of table 2 we compare units on the main diagonal. If the population would only include never-movers (that is if units were not sorting), we could attribute the difference between treatment and control clusters to the exposure. Yet, the presence of the other mover types complicate matters.

The second contrast is the difference between unexposed units and exposed units among the always-movers. While this contrast also compares exposed and unexposed units, the direction of the comparison is flipped. Assuming that treatment effects are homogeneous, this second contrast is the treatment effect but with the opposite sign.

The third contrast is not a causal contrast but a comparison of observed outcomes among the escapees (of which some were exposed while others were not) and followers (of which some are exposed and some or not). This last difference characterizes the heterogeneity in outcomes between units that move because of the exposure.

\section{Random Sorting}

Next, we outline three restrictive assumptions to ensure that sorting bias has the same consequences as non-differential measurement-error bias, which attenuates the average treatment effect toward zero (Lewbel, 2007).

We show that sorting dilutes the average treatment if sorting i) is not sparked by the exposure (A2, no escapees and followers), ii) has no causal effect on the outcome (A3, incidental sorting) and iii) types are homogeneous in their potential outcomes (A4, nondifferential types). Under these three assumptions, the average treatment effect is attenuated toward zero (diluted) as some units sort regardless of the exposure (the always-mover).

Formally,we write these three assumptions as follows:

Assumption 2 No escapees and followers

$M(1)=M(0)$

Assumption 3 Incidental sorting

$Y(0,1)=Y(1,1)$

$Y(0,0)=Y(1,0)$

Assumption 4 Partial non-differential mover types

$E[Y(1) \mid M=1]=E[Y(1) \mid M=0]$

$E[Y(0) \mid M=1]=E[Y(0) \mid M=0]$

The corresponding causal graph appears in Figure 2. 


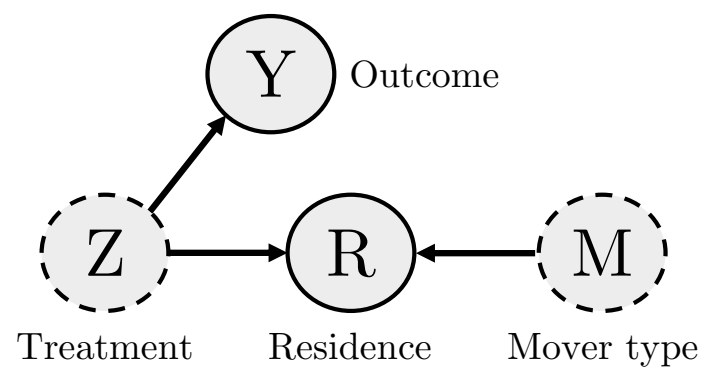

Figure 2: Directed acyclic graph of observed (solid) and unobserved (dashed) random variables under the random sorting assumptions (A1-A4).

The first assumption states that the exposure does not spark sorting. This is equivalent to say that there are no escapees or followers but only never- and always-movers. One implication of this assumption is that the realized movement indicator is sufficient to characterize units' types, that is, $M(0)=M(1)=M$. In other words, if $M_{i}=1$, this unit is an always-mover while the unit is a never-mover if $M_{i}=0$.

The incidental sorting assumption (A3) rules out any effect of moving on units' outcomes if these units have the same exposure status. An implication of this assumption is that we can focus on two instead of four potential outcomes: One for the unit when exposed, $Y_{i}(1)$, and one for the unit when it was not exposed, $Y_{i}(0)$. Complementarily, let $Y_{i}$ be the realized outcome which is either $Y_{i}(1)$ when the unit was exposed and $Y_{i}(0)$ otherwise.

In combination with the previous two assumptions, the non-differential mover type assumption rules out any heterogeneity between the average potential outcomes of never- and always-movers. We assume that their average potential outcomes under treatment (and under control) are identical. A stronger but unnecessary version of this assumption is to assume that all types are non-differential $(Y(1), Y(0) \Perp S$ or $Y(1), Y(0) \Perp M(1), M(0))$.

Combining all three assumptions (A2-A4) and maintaining the randomization assumption (A1), we have:

$$
\delta^{\prime}=\mathbb{E}[Y(1)-Y(0)] \underbrace{(p(Z=1 \mid R=1)-p(Z=1 \mid R=0))}_{\text {attenuation term }}=\delta \pi
$$

The proof appears in appendix C.1. Under the assumptions A0-A4, $\delta^{\prime}$ is the product of $\delta$ and a constant $\pi$ which is bounded between -1 and 1 .

In most instances, it is reasonable to assume that it is more likely to observe exposed rather than unexposed units in treatment clusters $(p(Z=1 \mid R=1)>0.5)$ and that it is more likely to observe unexposed rather than exposed units in control clusters $(p(Z=1 \mid R=$ $0)<0.5)$. If so, $\pi$ is bounded between 0 and 1 . 
The result highlights that even if the exposure does not spark movement, sorting is incidental and mover types are non-differential in their potential outcomes, there is still a mixing of exposed and unexposed units in treatment and control clusters. This mixing dilutes the ATE. In the most extreme scenario, when there is complete mixing (it is equally likely to observe exposed and unexposed units in treatment and control clusters), $\pi$ sets $\delta$ to 0 . In more moderate scenarios, the average treatment effect is attenuated toward 0 . If the joined distribution of $p(Z, R)$ (or $p(M, R)$ ) is known (e.g., from census tabulations), one could correct for this bias by dividing the observed difference between historically exposed and unexposed places $\left(\delta^{\prime}\right)$ by $\pi$.

The availability of census tabulations brings other opportunities. Suppose census data allow us to verify that $p(Z=1)=p(R=1)$, i.e., sorting does not alter the relative population distribution between exposed and unexposed places. In that case, A1-A3 are sufficient to show that $\delta^{\prime}=\mathbb{E}[Y(1)-Y(0)]-2 p(M=1) \mathbb{E}[Y(1)-Y(0) \mid M=1]$ (the proof appears in appendix C.1.1). This result shows that incidental sorting without escapees and followers and a stable relative population distribution leads to a bias that is additive and equals the product of the causal effect among the always-movers scaled by the size of the always-mover subpopulation.

\section{Non-Random Sorting}

While all three assumptions (A2-A4) are highly restrictive, the assumption that there are no escapees and followers appears in particular implausible. In the context of the US civilrights protest study, it amounts to assuming that no White residents moved from protest to non-protest counties because of the protest. Yet, a larger literature starting with Duncan and Duncan (1957) and (Taeuber and Taeuber, 1965) suggests that the arrival of Blacks in historically White neighborhoods sparked White out-migration. Since the protest increased the salience of the Black community in protest counties, one might suspect that the civilrights protest could have had a similar effect on Whites.

Relaxing the assumption of no escapees and followers (A2) and combining the remaining assumptions, we can write $\delta^{\prime}$ as follows:

$$
\begin{gathered}
\delta^{\prime}=\mathbb{E}[Y(1)-Y(0)] \underbrace{(p(Z=1 \mid R=1)-p(Z=1 \mid R=0))}_{\text {attenuation term }}+ \\
\underbrace{(p(S=F) \mathbb{E}[Y \mid S=F]-p(S=E) \mathbb{E}[Y \mid S=E]))}_{\text {sorting heterogeneity }}
\end{gathered}
$$


This result demonstrates that the difference between treatment and control clusters $\left(\delta^{\prime}\right)$ is the sum of an attenuated estimate of the average treatment effect (as discussed above) and an additive bias term that we refer to as sorting heterogeneity. The proof appears in appendix C.2.

The sorting heterogeneity is the observed difference describing the heterogeneity between two different subpopulations rather than a causal effect. Note that the resulting contrast compares two weighted means (but the weights do not sum to 1). The weighting makes the substantive interpretation of this contrast difficult in practice. However, suppose that the outcome is (on average) identical across the two subpopulations (this amounts to assuming that all types are non-differential, see above). Despite the absence of any heterogeneity, differences in the size of the follower and escapee subpopulation produce an observed difference. In fact, if the share of followers is larger than the share of escapees, the bias due to the sorting heterogeneity is positive while it is negative if there are more escapees than followers.

Consistent with the White-flight hypothesis, we might assume that there are escapees but no followers which means that the bias due to the sorting heterogeneity is negative. This assumption amounts to a monotonicity assumption, stipulating that the exposure either has no effect on a unit or a strictly positive effect (but not a negative effect). While such a monotonicity assumption has been used in other contexts - such as instrumental variable estimation (Angrist et al. 1996) — it seems plausible that there are at least some units living in non-protest counties that decided to move to protest counties because of the protests. In other words, it seems plausible that some Whites were attracted by the protests, as they signaled, for example, openness and tolerance.

The previous discussion assumes that the outcomes of escapees and followers are on average identical. What if they are not? In the context of the civil-rights protest study, it seems plausible to assume that there is outcome heterogeneity among followers and escapees: Those that left counties because of the protests (escapees) might harbor more racial resentment against Blacks today as compared with those that moved to these counties. Assuming that the two subpopulations are of equal size such heterogeneity in outcomes leads to a negative additive bias. If the two subpopulations differ in size and there are more escapees than followers, the magnitude of the bias increases.

With non-random sorting, we can not comfort ourselves that we merely underestimate the treatment effect as in a setting with random sorting. The key concern for applied research is that the true exposure effect might be zero and all differences between treatment and control clusters are pure sorting heterogeneity. Since the bias due to the sorting het- 
erogeneity is additive, observed differences between treatment and control clusters provide little information even about the sign of the exposure effect.

\section{Removing Sorting Bias by Design}

The previous discussion highlights that sorting bias is a threat to causal inference. The solution is, from a theoretical perspective, simple: Using data on people's exposure status instead of relying on current residency as a proxy avoids sorting bias. In practice, the best option is to construct an exposure variable based on information where respondents lived at the time of the treatment. We illustrate this strategy by reexamining the civilrights protest study of Mazumder (2018).$^{6}$ An alternative approach is to directly elicit respondents' exposure status. This alternative approach is only feasible when respondents are aware of their exposure status and can be expected to truthfully reveal it. An example of this approach is the study by Lupu and Peisakhin (2017).

One concern with the suggested solution is that survey responses come with measurement error which also biases estimates. It is therefore important to construct survey instruments in a way that they minimize the measurement error. For this reason, it might be useful to not elicit respondents' exposure status but to use information on respondents' place of residence in the past to construct their exposure status. In order to avoid measurement error altogether, it would be necessary to have validated information on respondents' place of residence or their exposure status from, perhaps, administrative data.

Our extended analysis uses data from the CCES 2010-2014 Panel Study Ansolabehere and Schaffner, 2015). This survey re-interviewed the 8014 White respondents from the CCES 2010 study in 2012 and 2014. Importantly, in 2012 respondents were asked in which town or city they lived when they were 17 years old and in 2014 in which town or city they lived when they were ten years old. Responses from these questions allow to reconstruct for a subset of White respondents in which county they lived between 1960 and 1965, and more specifically if they lived in a county where protests took place. The analysis sample includes White respondents and excludes everyone that did not live in the contiguous United States during the times of the protests $(N=3,244)$. A description of the survey items and how we identified respondents' county of residence can be found in appendix A.

We follow the original study for the baseline identification strategy and assume that the treatment exposure (protests) is as-if randomly assigned on the county-level, conditional on a series of covariates. The relevant covariates include: the average democratic vote share between 1932 and 1960, the logarithm of the county population as measured in 1960, median

\footnotetext{
${ }^{6}$ See $\operatorname{Biggs}$ et al. $(2020)$ for another discussion on this study.
} 
income 1960, the percentage figure of Blacks among the total population in 1960, as well as the percentage in urban areas in 1960. For a few respondents no information on their place of residency in the 1960s is available which reduces the number of observations in the regressions below.

The main outcome is a continuous, positive summary score from a principal component analysis (PCA) of the average response across three panel waves of the survey items used in the study. $]^{7}$ Higher values on this variable indicate more resentment toward Blacks (the item correlations are reported in Table SM.2). We prefer the PCA score, as it reduces measurement error and makes the presentation of the results more concise. However, in the appendix, tables SM.3 SM.6 we also report all regressions on each survey item separately.

\begin{tabular}{rcr}
\hline & \multicolumn{2}{c}{ Lived in protest } \\
Living in & county & $1960-65 ?$ \\
protest county & No & Yes \\
\hline No & 0.38 & 0.24 \\
Yes & 0.11 & 0.27 \\
\hline
\end{tabular}

Table 3: Proportion of White respondents by residency at the time of the survey (2012/2014) and place of residence at the time of the protests (1969-65). $N=3,244$.

Table 3 shows the number of respondents living in protest counties during 1960s and where they live today. We see that about $24 \%$ the respondents living in a protest county at the time of the protest moved to a non-protest county at the time of the survey. On the other hand, about $11 \%$ of the respondents did not live in a protest county but live in such a county now. Overall, we see that about a third of the respondents moved from protest to non-protest counties (and vice versa). While we cannot distinguish if the movers are alwaysmovers, escapees or followers, it is obvious that there is ample room for post-treatment sorting bias.

Table 4 (column 1) replicates the baseline finding using the full sample 8 Respondents living in counties that were the scene of civil rights protests in 1960-65 are much less resentful toward Blacks than those living counties were no such protests occurred. The estimated effect suggests that respondents living in a protest county have a 0.14 lower PCA score than those living elsewhere. This effect is about $7.8 \%$ of a standard deviation in the PCA score.

\footnotetext{
${ }^{7}$ To allow for a concise discussion of the results below, we construct the PCA score to be strictly positive by shifting all values with some constant.

${ }^{8}$ In the appendix, I present estimates based on county-level aggregates following the original study (see Table SM.7). I also demonstrate that the estimates are very similar when using the provided survey weights (see Table SM.8.
} 


\begin{tabular}{|c|c|c|c|c|c|}
\hline & \multicolumn{4}{|c|}{ Resentment PCA Score } & \multirow{2}{*}{$\frac{\text { Stays? }}{\text { Model } 5}$} \\
\hline & Model 1 & Model 2 & Model 3 & Model 4 & \\
\hline Lives in protest county? & $\begin{array}{l}-0.14^{*} \\
(0.08)\end{array}$ & $\begin{array}{c}-0.28^{* * *} \\
(0.10)\end{array}$ & $\begin{array}{c}-0.30^{* * *} \\
(0.07)\end{array}$ & & \\
\hline $\begin{array}{l}\text { Lived in protest county } \\
1960-65 ?\end{array}$ & & & & $\begin{array}{l}-0.00 \\
(0.10)\end{array}$ & $\begin{array}{c}-0.23^{* * *} \\
(0.03)\end{array}$ \\
\hline $\mathrm{N}$ & 7929 & 3212 & 3216 & 3216 & 3216 \\
\hline $\begin{array}{l}\text { Residence county } \\
\text { State FE } \\
\text { Covariates }\end{array}$ & $\begin{array}{l}\text { Yes } \\
\text { Yes }\end{array}$ & $\begin{array}{l}\text { Yes } \\
\text { Yes }\end{array}$ & $\begin{array}{l}\text { No } \\
\text { No }\end{array}$ & $\begin{array}{l}\mathrm{No} \\
\mathrm{No}\end{array}$ & $\begin{array}{l}\text { No } \\
\text { No }\end{array}$ \\
\hline $\begin{array}{l}\text { Residence county 1960-65 } \\
\text { State FE } \\
\text { Covariates }\end{array}$ & $\begin{array}{l}\text { No } \\
\text { No }\end{array}$ & $\begin{array}{l}\text { No } \\
\text { No }\end{array}$ & $\begin{array}{l}\text { Yes } \\
\text { Yes }\end{array}$ & $\begin{array}{l}\text { Yes } \\
\text { Yes }\end{array}$ & $\begin{array}{l}\text { Yes } \\
\text { Yes }\end{array}$ \\
\hline
\end{tabular}

Table 4: OLS regression estimates with cluster-robust standard errors at the county level. The dependent variable is the racial resentment summary score from a principal component of four survey items measured in 2010-2014 (col. 1-4) and an indicator whether a respondent lived in a protest (non-protest) county at the time of the protests and at the time of the survey (col. 5). Higher values indicate more racial resentment. The sample includes all CCES respondents (col. 1) and all CCES respondents that were ten or 17 years old in 196065 when the protests took place (col. 2-5).

The second column of the same table replicates the baseline results for the sample of respondents for which information about their place of residence 1960-65 is available. The estimated effect in this subsample is larger suggesting that there is effect heterogeneity by birth cohort. In the third column, we report the estimates from a regression where instead of including state fixed-effect for respondents' current county of residence and covariates about the attributes of their current county of residence, we include a fixed-effect for the state of the county respondents lived in and covariates on the attributes of their county of residence in the 1960s. This adjustment is necessary to remove some of the post-treatment bias that comes from conditioning on post-treatment covariates. The estimated effect is a bit larger again.

The fourth column includes an indicator if the respondent lived in a protest county during the time of the protests instead of an indicator if the respondent now lives in a protest county. In this specification the estimated effect is zero and the standard error is about 0.1. This means that respondents who experienced protests during 1960-65 have about the same level of racial resentment toward Blacks today as respondents that did not experience the protests 
in 1960-65. The estimate in the fourth column suggests that much of the differences between respondents living and not living in protest counties now are due to post-treatment sorting $!^{9}$

The estimate in the fourth column is also consistent with another interpretation: The indicator for protest exposure in the 1960s was measured with error and, therefore, the estimate of the effect is attenuated to zero. There are two reasons that speak against this interpretation. First, the indicator is not based on respondents' self-reported exposure to the protests but based on their reported place of residence during adolescence. Although retrospective survey questions always come with the risk of recall error, reporting one's place of residence during adolescence seems less demanding than recalling protest exposure. Second, if the indicator for protest exposure in the 1960s was noise alone, we would expect to never find any effects. However, the next result shows that this is not the case.

The fifth column shows that the protests were a strong push factor for Whites. In the last column of table 4, we regress an indicator if a respondent lived in the same type of county between 1960 and 1965 and in 2010 (either always in a treatment county or always in a control county) on the indicator for protests in 1960-65. The estimated coefficient suggests that the chances to remain in the same county-type is about $23 \%$ lower for those that lived in protest counties in 1960-65, as compared with those in the non-protest counties. This suggests that the protests induced White flight. However, it is important to keep in mind that this result only means that there are relatively more escapees (White fleeing from protest counties) than followers (White attracted to move to protest counties).

The results demonstrate that the sorting heterogeneity (the second under-braced term in equation (8) is negative. This is consistent with the hypothesis articulated in the previous section that the respondents who left protest counties because of the protests (the escapees) have on average higher resentment toward Blacks as compared with those that moved to these counties (the followers). The fact that there are more escapees than followers amplifies the difference and therefore increases the magnitude of the negative bias.

\section{Existing Strategies to Address Sorting}

Most legacy studies, including the civil-rights protest study, grapple with sorting in one way or another. Depending on data availability, legacy studies use three different strategies to address sorting. The first strategy is to evaluate if migration flows are differential between historically exposed and unexposed places. In the absence of any differences, authors argue

\footnotetext{
${ }^{9}$ The absence of an average treatment effect may mask treatment effect heterogeneity by latent mover type. In principle, it is possible that there is a positive treatment effect among some of the types (e.g., the nevermovers) and a negative treatment effect among any of the other types (e.g., the escapees). We leave it to future research to devise a means to estimate treatment effect heterogeneity by latent mover type.
} 
that sorting is not a concern. From the perspective of the proposed framework, we can think of this strategy as seeking to estimate the share of escapees and followers. Their presence is a necessary condition for a bias due to sorting heterogeneity. Estimating the share of escapees and followers is feasible if data on in-migration and out-migration are available. However, in practice, authors often only have access to data on net-migration (in-migration minus out-migration), which is only partially informative because a non-differential net-migration may mask an equally sized but sizeable share of escapees and followers. A case in point is the example in Figure 1, where net-migration is exactly zero, but sorting remains a concern. The civil-rights protest study documents that the White net-migration rate based on US Census records were slightly lower in protest counties relative to non-protest counties during the 1960s and the 1970s (the difference is only statistically significant in the 1970s). This is consistent with the finding from the analysis in the previous section that there are more escapees relative to followers. However, different from this analysis, the pattern documented in the civil-rights protest study is limited to the two decades after the protests. Even if the net-migration rates were exactly equal during these two decades, there would still be ample room for sorting up until when outcomes are actually measured.

The second strategy used by legacy studies to address sorting is to conduct a mediation analysis using the data on migration rates. Prompted by the statistical significant differences in net-migration rates during the 1970s, the author of the civil-rights protest study uses this strategy. The results of the causal mediation analysis (Imai et al., 2011, 2010) indicate that the average direct effect (ADE) equals almost exactly the estimated treatment treatment. The author then concludes: "As a result, it does not seem that sorting alone can explain the entirety of the results." That conclusion, however, is premature. The estimated ADE describes the effect of the protests when the net-migration rate was held constant at the value that would have been realized in a world without protests. In other words, the ADE describes how a community-level variable mediates the effect of the exposure. Although the absence of the estimated mediation effect is an interesting result on its own, it does not help to isolate the average causal effect from the bias that comes with the units' own sorting behavior. It is, of course, equally premature to conclude that mediation analysis is ineffective for all legacy studies. For cluster-level legacy studies, mediation analysis delivers the relevant causal quantity as we discuss in the next section.

A third and final strategy used by legacy studies to address sorting is to use rich census data to compare correlates of political behavior and attitudes of movers and non-movers. An example of this approach is the study by Acharya et al. (2016), who show that contemporary racial resentment toward Blacks and opposition to affirmative action in Southern US counties goes back to the prevalence of slavery 150 years ago. For data availability reasons, they 
study the demographics of movers and non-movers in the 1930 US Census, writing: "If sorting plays an important role in our results, we would expect to see differences between migrants to/from high-slave areas versus low-slave areas" (p. 630). In terms of Table 2, we can think of their comparison as contrasting the difference between movers (the off-diagonal cells) versus non-movers (the diagonal cells) in each column. However, since each cell in the table is populated by two distinct unobserved types, their contrast does not isolate a (relative) difference between any of the four mover types. Although this does not invalidate their analysis, the absence of any difference does not imply that there is no post-treatment sorting, which originates in outcome differences between the four mover types. If it were credible to assume that one of the four mover types does not exist, one could use ideas from complier profiling in instrumental variable estimation to characterize the remaining three types (Marbach and Hangartner, 2020). However, as discussed above, invoking a monotonicity assumption seems difficult to justify.

\section{Extensions to Other Settings}

There are a large variety of legacy studies. Surveying all legacy studies recently reviewed by (Cirone and Pepinsky, 2021), we find that the overwhelming majority of legacy studies examines exposures that vary across geography (about 86\%). Among these studies, about a quarter focus on the effect of a one-time shock on a unit's own outcomes - the setting we focus on in this paper. In this discussion section, we discuss the role of sorting in other settings, including settings in which the exposure is permanent rather than a one-time shock, exposure-outcome effects spanning generations and outcomes that are measured on the cluster, rather than the individual level. The discussion highlights that sorting is not less important in these settings.

Some legacy studies focus on exposure effects on a unit's descendants rather than on a unit's own outcomes. While the framework presented in this paper does not directly apply, the obstacles in identifying causal effects in such a setting are similar because a person's ancestors also have agency about their location choices. Empirically, the main difficulty is to track residence patterns for someone's multiple ancestors 10 Theoretically, the difficulty is to define how the exposure status of a person's multiple ancestors maps into the exposure status of an individual.

\footnotetext{
${ }^{10}$ Multi-generational surveys are rare but not unheard of in the literature on legacy studies. For example, Lupu and Peisakhin (2017) conduct a multi-generational survey to show that that "descendants of individuals who suffered more intensely [from the deportation of Crimean Tatars in 1944] identify more strongly with their ethnic group, support more strongly the Crimean Tatar political leadership, hold more hostile attitudes toward Russia, and participate more in politics."
} 
In the one-time shock setting we focus on, units' assigned exposure is identical to units' exposure intake. Therefore, units are deprived of their ability to select their exposure after assignment by moving. For example, in the one-time-shock setting, followers moving to exposed places remain unexposed and escapees moving to unexposed places remain exposed. However, in some settings, units have the ability to select their exposure intake (or exposure length) by moving after assignment.11 In settings in which the exposure is permanently changing institutions, policies, or socioeconomic features, units' agency over their own exposure intake via moving further complicates the identifiability of the exposure-outcome effect. From a practical perspective, this means that identifying the legacy effects of, for example, institutional change (a permanent exposure) may be more difficult as compared with legacy effects of an event (a one-time shock).

The setting we consider is one in which the causal estimand is the difference in a unit's potential outcome when exposed and a unit's potential outcome when not exposed. However, as already pointed out earlier, not all legacy studies target this quantity. What is the role of sorting in legacy studies focusing on the effects of an exposure on cluster-level outcomes rather than individual-level outcomes? In cluster-level legacy studies, the estimand is the difference between a cluster's potential outcome when exposed and a cluster's potential outcome when not exposed. Under random assignment of the exposure, these cluster-level differences are causally identified. Although cluster-level randomization of the exposure is sufficient to identify the exposure-outcome effect, the identification challenge in these studies is to discern if there is a causal effect net of the exposure effect on sorting.

We can cast the identification challenge in cluster-level legacy studies in terms of a causal mediation problem. Let $Y_{j}^{\prime}(o, n, z)$ be the potential outcome for a cluster $j$ with exposure $z$, out-migration level $o$, and in-migration level $n$. We also define potential out-migration and in-migration levels for cluster $j$ given exposure $z$, i.e., $O_{j}(z)$ and $N_{j}(z)$. Under random assignment, the average causal effect (total effect), i.e., $\mathbb{E}\left[Y^{\prime}(O(1), N(1), 1)-Y^{\prime}(O(0), N(0), 0)\right]$, is identifiable. The challenge is to identify the average natural direct effect (NDE), i.e., $\mathbb{E}\left[Y^{\prime}(O(0), N(0), 1)-Y^{\prime}(O(0), N(0), 0)\right]$, which describes the effect of the exposure on clusterlevel outcomes if out- and in-migration were set to the their level without the exposure. A growing literature discusses the causal identification assumption for NDEs that often involve cross-world comparisons (see, for example, Imai et al., 2011, 2010).

\footnotetext{
${ }^{11}$ In these settings, the exposure is a function of the latent mover type, i.e., $Z(S)$.
} 


\section{Conclusion}

How much does history shape contemporary political behavior and attitudes? A number of studies suggest that the answer is "a lot." However, at the heart of these studies is often a comparison between places that were historically exposed to some treatment versus those that were not. As we demonstrate in this paper, the observed differences can only be interpreted as a causal effect if a number of strong identifying assumptions are invoked about how units migrate between historically exposed and unexposed places. If these identifying assumptions are not met, estimates will be contaminated by post-treatment sorting bias.

The fundamental problem of causal inference is that counterfactuals can never be observed. From this perspective, post-treatment sorting is a much smaller problem as the bias can be ruled out by design in principle with information about units' historic exposure status. This suggests that the best course of action for applied research is to find and collect better data. As demonstrated in this study, information about where survey respondents lived in the past is critical to obtain reliable estimates about how history shapes political behavior and attitudes.

The results in this paper may help to guesstimate the magnitude and direction of the bias. One critical take-away point is that even in the best case scenario (random sorting), we should expect the treatment effect to be biased toward zero because the presence of alwaysmovers dilutes the treatment effect. Moreover, the longer the period between treatment and outcome measurement, the higher the attenuation bias. As time passes, more and more units will move regardless of their exposure status (the share of always-movers is increasing). This means that the attenuation of the treatment effect should be expected to grow as time passes. Therefore, we may be in particular skeptical about studies demonstrating that the causal effects are dynamically increasing as time passes.

When using the results presented in this paper to guesstimating the magnitude and direction of the bias, it is important to defend the underlying assumptions. That might not always be easy. For example, defending the incidental sorting assumption is difficult as the literature on the effects of moving on political behavior and attitudes in advanced democracies is rather thin and conflicting. While some studies suggest that movers adopt the political ideology of their neighbors (e.g., Gallego et al., 2016), others find no effects on immigration attitudes among movers to urban areas (e.g., Maxwell, 2019). Legacy studies would clearly benefit from more research on how domestic migration shapes, and is shaped by, political behavior and attitudes.

Another lesson from this paper is that there are advantages of studying the effects of history with exposures that vary across groups rather than geography. Assuming that group membership is not subject to units' choice, the type of post-treatment bias outlined in this 
paper is avoided. An example of this approach is the paper by Nunn and Wantchekon (2011) who study the effect of the slave trade on trust in Africa. Using contemporary survey data, their main analysis avoids the type of post-treatment sorting bias described in this paper because the authors collected data on the intensity of the slave trade by ethnic group rather than (only) by geography. 


\section{References}

Acharya, A., M. Blackwell, and M. Sen (2016). The Political Legacy of American Slavery. The Journal of Politics 78(3), 621-641.

Aigner, D. J. (1973). Regression with a Binary Independent Variable Subject to Errors of Observation. Journal of Econometrics 1(1), 49-59.

Angrist, J. D., G. W. Imbens, and D. B. Rubin (1996). Identification of Causal Effects Using Instrumental Variables. Journal of the American Statistical Association 91(434), 444-455.

Ansolabehere, S. and B. Schaffner (2015). Cooperative Congressional Election Study: 2010-2014 Panel Study. Data Release No. 2. Available at https://doi.org/10.7910/DVN/TOE8I1.

Barceló, J. (2021). The Long-Term Effects of War Exposure on Civic Engagement. Proceedings of the National Academy of Sciences 118(6).

Biggs, M., C. Barrie, and K. T. Andrews (2020). Did Local Civil Rights Protest Liberalize Whites' Racial Attitudes? Research \& Politics 7(3), 2053168020914757.

Brown, J. R. and R. D. Enos (2021). The Measurement of Partisan Sorting for 180 Million Voters. Nature Human Behaviour, 1-11.

Cantoni, E. and V. Pons (2022). Does Context Trump Individual Drivers of Voting Behavior? Evidence from U.S. Movers. American Economic Review 112(4), 1226-72.

Cirone, A. and T. B. Pepinsky (2021). Historical persistence. Annual Review of Political Science 25.

Duncan, O. D. and B. Duncan (1957). The Negro Population of Chicago: A Study of Residential Succession. Chicago: University of Chicago Press.

Frangakis, C. E. and D. B. Rubin (2004). Principal Stratification in Causal Inference. Biometrics 58(1), 21-29.

Gallego, A., F. Buscha, P. Sturgis, and D. Oberski (2016). Places And Preferences: A Longitudinal Analysis of Self-selection and Contextual Effects. British Journal of Political Science 46(3), 529-550.

Homola, J., M. M. Pereira, and M. Tavits (2020). Legacies of the Third Reich: Concentration Camps and Out-Group Intolerance. American Political Science Review 114(2), 573-590. 
Imai, K., L. Keele, D. Tingley, and T. Yamamoto (2011). Unpacking the Black Box of Causality: Learning about Causal Mechanisms from Experimental and Observational Studies. American Political Science Review 105(4), 765-789.

Imai, K., L. Keele, and T. Yamamoto (2010). Identification, Inference and Sensitivity Analysis for Causal Mediation Effects. Statistical science 25(1), 51-71.

Imai, K. and T. Yamamoto (2010). Causal Inference with Differential Measurement Error: Nonparametric Identification and Sensitivity Analysis. American Journal of Political Science 54(2), 543-560.

Kelly, M. (2019). The Standard Errors of Persistence. Unpublished.

Lewbel, A. (2007). Estimation of Average Treatment Effects with Misclassification. Econometrica $75(2), 537-551$.

Lupu, N. and L. Peisakhin (2017). the Legacy of Political Violence across Generations. American Journal of Political Science 61(4), 836-851.

Marbach, M. and D. Hangartner (2020). Profiling Compliers and Non-compliers for Instrumental Variable Analysis. Political Analysis 28(3), 435-444.

Maxwell, R. (2019). Cosmopolitan immigration attitudes in large european cities: Contextual or compositional effects? American Political Science Review 113(2), 456-474.

Mazumder, S. (2018). The Persistent Effect of US Civil Rights Protests on Political Attitudes. American Journal of Political Science 62(4), 922-935.

Nunn, N. and L. Wantchekon (2011). The Slave Trade and the Origins of Mistrust in Africa. American Economic Review 101(7), 3221-52.

Rozenas, A. and Y. M. Zhukov (2019). Mass Repression and Political Loyalty: Evidence From Stalin's 'Terror by Hunger'. American Political Science Review 113(2), 569-583.

Taeuber, K. E. and A. F. Taeuber (1965). Negroes in Cities: Residential Segregation and Neighborhood Change. Chicago: Aldine Publishing.

Voth, H.-J. (2021). Persistence-Myth and Mystery. In The Handbook of Historical Economics, pp. 243-267. Elsevier. 


\section{Supplementary Information: \\ Causal Effects, Migration and Legacy Studies}

\section{Contents}

\begin{tabular}{lr}
\hline References & 23
\end{tabular}

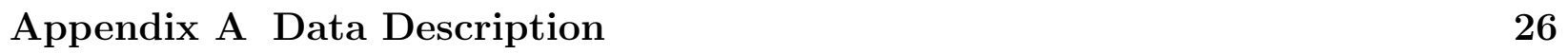

\begin{tabular}{|lll}
\hline Appendix B & Further Empirical Results & 29
\end{tabular}

\begin{tabular}{|l|l}
\hline Appendix C Proofs & 34
\end{tabular}

C.1 Random Sorting . . . . . . . . . . . . . . . . . . . . . . . . . . . 34

C.1.1 Random sorting with a stable population distribution . . . . . . . . 35

C.2 Non-Random Sorting . . . . . . . . . . . . . . . . . . . . . . . . . 36 


\section{Appendix A Data Description}

The data come from the 2010-2014 Cooperative Congressional Election Study Panel Survey $(N=9500)$. Following the Mazumder $(2018)$ we subset to White respondents $(N=8014)$. The outcome variable in the main text, the summary score for racial resentment, comes from a principal component analysis (PCA). The PCA is run on the average response across the three panel waves for the following four items:

- Affirmative action item: "Affirmative action programs give preference to racial minorities in employment and college admissions to correct for past discrimination. Do you support or oppose affirmative action?" (Strongly support, Somewhat support, Somewhat oppose, Strongly oppose)

- Racial resentment item 1: "The Irish, Italians, Jews, and many other minorities overcame prejudice and worked their way up. Blacks should do the same without any special favors." (Strongly agree, Somewhat agree, Neither agree nor disagree, Somewhat disagree, Strongly disagree)

- Racial resentment item 2: "Generations of slavery and discrimination have created conditions that make it difficult for Blacks to work their way out of the lower class." (Strongly agree, Somewhat agree, Neither agree nor disagree, Somewhat disagree, Strongly disagree)

- Binary indicator if someone identifies as Democrat based on the question "Generally speaking, do you think of yourself as a ... ?" (Democrat, Republican, Independent, Other)

Two survey respondents with missing survey responses are excluded from the PCA. The correlations between the items and the PCA score are reported in Table SM.2.

In the appendix, separate regressions for three outcomes as in the original study are reported:

- Racial resentment: The average of the response to the first racial resentment item (reversed coding) and the answer to the second racial resentment item (averaged across three survey waves).

- Affirmative action: Binary indicator if respondent supports/somewhat supports affirmative action (averaged across the three survey waves).

- Democratic identification: Binary indicator if someone identifies as Democrat (averaged across the three survey waves). 
The following items are used to measure county of residence between 1960 and 1965 for a subset of respondents born between 1943-1948 and 1950-1955.

- "In what town or city did you live on your 17th birthday?"

(open-ended question, only asked in 2012)

- "In what state did you live on your 17th birthday?"

(dropdown menu, asked in 2012)

- "In what country did you live on your 17th birthday?"

(dropdown menu, asked in 2012)

- "In what town or city did you live on your 10th birthday?"

(open-ended question, only asked in 2014)

- "In what state did you live on your 10th birthday?"

(dropdown menu, asked in 2014)

- "In what country did you live on your 10th birthday?"

(dropdown menu, asked in 2014)

Excluding all respondents reporting to not have lived in the United States, we use the Google Maps API to geocode respondents using the API query string "[Town or city], [State], USA". Using the API provided longitude and latitude, we assign a county identifier (FIPS) to everyone that lives in the contiguous United States (this excludes Alaska, Hawaii and Territories of the United States) using a GIS county map. A few respondents could not be located (reported in Table SM.1 as "unmatched") because they did not provide information in which town/city or state they lived or the information they provided was inconsistent (e.g., the town/city was located in a state that is different from their reported state). 


\begin{tabular}{|c|c|c|c|c|}
\hline Age & Location & Match? & $\mathrm{N}$ & in $\%$ \\
\hline \multirow[t]{8}{*}{17} & USA (contiguous) & & 9191 & 96.75 \\
\hline & & Yes & 9075 & 98.74 \\
\hline & & No & 116 & 1.26 \\
\hline & & Total & 9191 & 100 \\
\hline & USA (other) & & 51 & 0.54 \\
\hline & Other country & & 228 & 2.40 \\
\hline & Unknown & & 30 & 0.32 \\
\hline & Total & & 9500 & 100 \\
\hline \multirow[t]{8}{*}{10} & USA (contiguous) & & 9068 & 95.45 \\
\hline & & Yes & 8907 & 98.22 \\
\hline & & No & 161 & 1.78 \\
\hline & & Total & 9068 & 100 \\
\hline & USA (other) & & 56 & 0.59 \\
\hline & Other country & & 310 & 3.26 \\
\hline & Unknown & & 66 & 0.69 \\
\hline & Total & & 9500 & 100 \\
\hline
\end{tabular}

Table SM.1: Number and proportion of respondents by location and age. For respondents located in the contiguous United States, the table reports if their information was sufficiently detailed to locate them in a particular county. 


\section{Appendix B Further Empirical Results}

\begin{tabular}{lccc}
\hline & Racial resent. & Affirm. action & Democrat \\
\hline PCA Score & $0.68^{* * *}$ & $-0.20^{* * *}$ & $-0.19^{* * *}$ \\
& $(0.00)$ & $(0.00)$ & $(0.00)$ \\
\hline Num. obs. & 8012 & 8012 & 8012 \\
$\mathrm{R}^{2}$ & 0.92 & 0.75 & 0.53 \\
\hline${ }^{* * *} p<0.01 ;{ }^{* *} p<0.05 ;{ }^{*} p<0.1$ & &
\end{tabular}

Table SM.2: OLS regression estimates for the three survey items and the PCA summary score.

\begin{tabular}{lcccc}
\hline & Racial resent. & Affirm. action & Democrat & PCA Score \\
\hline Lives in protest county? & -0.07 & $0.04^{*}$ & $0.04^{* *}$ & $-0.14^{*}$ \\
& $(0.06)$ & $(0.02)$ & $(0.02)$ & $(0.08)$ \\
Percent Black & -0.00 & 0.00 & 0.00 & -0.01 \\
& $(0.00)$ & $(0.00)$ & $(0.00)$ & $(0.00)$ \\
Percent Urban & $-0.00^{*}$ & 0.00 & $0.00^{* *}$ & $-0.00^{* *}$ \\
& $(0.00)$ & $(0.00)$ & $(0.00)$ & $(0.00)$ \\
$\log$ (Population) & -0.04 & 0.01 & 0.00 & -0.05 \\
Median Income & $(0.03)$ & $(0.01)$ & $(0.01)$ & $(0.04)$ \\
& -0.00 & 0.00 & -0.00 & -0.00 \\
Avg. Dem. Vote Share & $(0.00)$ & $(0.00)$ & $(0.00)$ & $(0.00)$ \\
& 0.34 & -0.02 & 0.08 & 0.35 \\
& $(0.22)$ & $(0.07)$ & $(0.08)$ & $(0.31)$ \\
\hline Num. obs. & 7931 & 7931 & 7931 & 7929 \\
Residence county & & & & \\
State FE & Yes & Yes & Yes & Yes \\
Covariates & Yes & Yes & Yes & Yes \\
\hline${ }^{* * *} p<0.01{ }^{* *} p<0.05,{ }^{*} p<0.1$ & & & &
\end{tabular}

Table SM.3: OLS regression estimates with cluster-robust standard errors at the county level separately for survey items (col. 1-3) and the PCA summary score (col. 4). 


\begin{tabular}{lcccc}
\hline & Racial resent. & Affirm. action & Democrat & PCA Score \\
\hline Lives in protest county? & $-0.16^{* *}$ & $0.06^{* *}$ & $0.08^{* * *}$ & $-0.28^{* * *}$ \\
& $(0.07)$ & $(0.02)$ & $(0.03)$ & $(0.10)$ \\
Percent Black & -0.00 & 0.00 & -0.00 & 0.00 \\
& $(0.00)$ & $(0.00)$ & $(0.00)$ & $(0.01)$ \\
Percent Urban & $-0.00^{* *}$ & 0.00 & 0.00 & $-0.01^{* *}$ \\
& $(0.00)$ & $(0.00)$ & $(0.00)$ & $(0.00)$ \\
$\log ($ Population) & 0.02 & -0.00 & -0.01 & 0.02 \\
Median Income & $(0.03)$ & $(0.01)$ & $(0.01)$ & $(0.05)$ \\
& -0.00 & 0.00 & -0.00 & 0.00 \\
Avg. Dem. Vote Share & $(0.00)$ & $(0.00)$ & $(0.00)$ & $(0.00)$ \\
& 0.26 & 0.05 & 0.08 & 0.23 \\
& $(0.28)$ & $(0.09)$ & $(0.11)$ & $(0.39)$ \\
\hline Num. obs. & 3212 & 3212 & 3212 & 3212 \\
Residence county & & & & \\
State FE & Yes & Yes & Yes & Yes \\
Covariates & Yes & Yes & Yes & Yes \\
\hline$* * * p<0.01,{ }^{* *} p<0.05,{ }^{*} p<0.1$ & & & &
\end{tabular}

Table SM.4: OLS regression estimates with cluster-robust standard errors at the county level separately for survey items (col. 1-3) and the PCA summary score (col. 4). 


\begin{tabular}{lcccc}
\hline & Racial resent. & Affirm. action & Democrat & PCA Score \\
\hline Lives in protest county? & $-0.17^{* * *}$ & $0.07^{* * *}$ & $0.09^{* * *}$ & $-0.30^{* * *}$ \\
& $(0.05)$ & $(0.01)$ & $(0.02)$ & $(0.07)$ \\
Percent Black & -0.00 & 0.00 & $-0.00^{* *}$ & 0.00 \\
& $(0.00)$ & $(0.00)$ & $(0.00)$ & $(0.01)$ \\
Percent Urban & -0.00 & 0.00 & 0.00 & -0.00 \\
& $(0.00)$ & $(0.00)$ & $(0.00)$ & $(0.00)$ \\
log(Population) & 0.01 & -0.00 & -0.01 & 0.02 \\
Median Income & $(0.03)$ & $(0.01)$ & $(0.01)$ & $(0.04)$ \\
& -0.00 & 0.00 & -0.00 & -0.00 \\
Democratic vote share & $(0.00)$ & $(0.00)$ & $(0.00)$ & $(0.00)$ \\
& 0.07 & 0.04 & $0.24^{*}$ & -0.17 \\
& $(0.31)$ & $(0.11)$ & $(0.13)$ & $(0.45)$ \\
\hline Num. obs. & 3216 & 3216 & 3216 & 3216 \\
Residence county $1960-65$ & & & & \\
State FE & Yes & Yes & Yes & Yes \\
Covariates & Yes & Yes & Yes & Yes \\
\hline${ }^{* * *} p<0.01,{ }^{* *} p<0.05,{ }^{*} p<0.1$ & & & &
\end{tabular}

Table SM.5: OLS regression estimates with cluster-robust standard errors at the county level separately for survey items (col. 1-3) and the PCA summary score (col. 4). 


\begin{tabular}{lcccc}
\hline & Racial resent. & Affirm. action & Democrat & PCA Score \\
\hline Lived in protest county 1960-65? & 0.00 & 0.01 & 0.01 & -0.00 \\
& $(0.07)$ & $(0.02)$ & $(0.02)$ & $(0.10)$ \\
Percent Black & -0.00 & 0.00 & $-0.00^{* *}$ & 0.00 \\
& $(0.00)$ & $(0.00)$ & $(0.00)$ & $(0.01)$ \\
Percent Urban & -0.00 & 0.00 & 0.00 & -0.00 \\
& $(0.00)$ & $(0.00)$ & $(0.00)$ & $(0.00)$ \\
$\log$ (Population) & 0.00 & 0.00 & -0.00 & -0.00 \\
Median Income & $(0.03)$ & $(0.01)$ & $(0.01)$ & $(0.05)$ \\
& -0.00 & 0.00 & -0.00 & -0.00 \\
Democratic vote share & $(0.00)$ & $(0.00)$ & $(0.00)$ & $(0.00)$ \\
Residence county 1960-65 & 0.07 & 0.04 & $0.24^{*}$ & -0.15 \\
State FE & $(0.31)$ & $(0.10)$ & $(0.12)$ & $(0.45)$ \\
Covariates & & & & \\
\hline
\end{tabular}

${ }^{* * *} p<0.01,{ }^{* *} p<0.05,{ }^{*} p<0.1$

Table SM.6: OLS regression estimates with cluster-robust standard errors at the county level separately for survey items (col. 1-3) and the PCA summary score (col. 4).

\begin{tabular}{|c|c|c|c|c|c|}
\hline & \multicolumn{4}{|c|}{ Resentment PCA Score } & \multirow{2}{*}{$\frac{\text { Stays? }}{\text { Model } 5}$} \\
\hline & Model 1 & Model 2 & Model 3 & Model 4 & \\
\hline Lives in protest county? & $\begin{array}{l}-0.08 \\
(0.10)\end{array}$ & $\begin{array}{c}-0.30^{* *} \\
(0.12)\end{array}$ & $\begin{array}{c}-0.24^{* * *} \\
(0.09)\end{array}$ & & \\
\hline $\begin{array}{l}\text { Lived in protest county } \\
1960-65 ?\end{array}$ & & & & $\begin{array}{l}-0.03 \\
(0.12)\end{array}$ & $\begin{array}{c}-0.26^{* * *} \\
(0.04)\end{array}$ \\
\hline $\mathrm{N}$ & 7929 & 3212 & 3216 & 3216 & 3216 \\
\hline \multicolumn{6}{|l|}{ Residence county } \\
\hline State FE & Yes & Yes & No & No & No \\
\hline Covariates & Yes & Yes & No & No & No \\
\hline \multicolumn{6}{|l|}{ Residence county 1960-65 } \\
\hline State FE & No & No & Yes & Yes & Yes \\
\hline Covariates & No & No & Yes & Yes & Yes \\
\hline
\end{tabular}

Table SM.7: Weighted OLS regression estimates with cluster-robust standard errors at the county level and survey weights. 


\begin{tabular}{|c|c|c|}
\hline & PCA Score & PCA Score \\
\hline Lives in protest county? & $\begin{array}{c}-0.28^{* *} \\
(0.14)\end{array}$ & \\
\hline Lived in protest county $1960-65 ?$ & & $\begin{array}{c}0.03 \\
(0.14)\end{array}$ \\
\hline Percent Black & $\begin{array}{c}0.01 \\
(0.01)\end{array}$ & $\begin{array}{l}-0.01 \\
(0.01)\end{array}$ \\
\hline Percent Urban & $\begin{array}{l}-0.00 \\
(0.00)\end{array}$ & $\begin{array}{c}0.00 \\
(0.00)\end{array}$ \\
\hline $\log ($ Population $)$ & $\begin{array}{c}0.00 \\
(0.07)\end{array}$ & $\begin{array}{l}-0.05 \\
(0.07)\end{array}$ \\
\hline Median Income & $\begin{array}{c}0.00 \\
(0.00)\end{array}$ & $\begin{array}{l}-0.00 \\
(0.00)\end{array}$ \\
\hline Democratic vote share & $\begin{array}{c}0.46 \\
(0.51)\end{array}$ & $\begin{array}{c}0.54 \\
(0.59)\end{array}$ \\
\hline Num. obs. & 1035 & 965 \\
\hline $\begin{array}{l}\text { Residence county } \\
\text { State FE } \\
\text { Covariates }\end{array}$ & $\begin{array}{l}\text { Yes } \\
\text { Yes }\end{array}$ & $\begin{array}{l}\text { No } \\
\text { No }\end{array}$ \\
\hline $\begin{array}{l}\text { Residence county 1960-65 } \\
\text { State FE } \\
\text { Covariates }\end{array}$ & $\begin{array}{l}\text { No } \\
\text { No }\end{array}$ & $\begin{array}{l}\text { Yes } \\
\text { Yes }\end{array}$ \\
\hline
\end{tabular}

Table SM.8: OLS regression estimates with heteroscedasticity-robust standard errors using county-level aggregates. 


\section{Appendix C Proofs}

\section{C.1 Random Sorting}

Using the incidental sorting assumption:

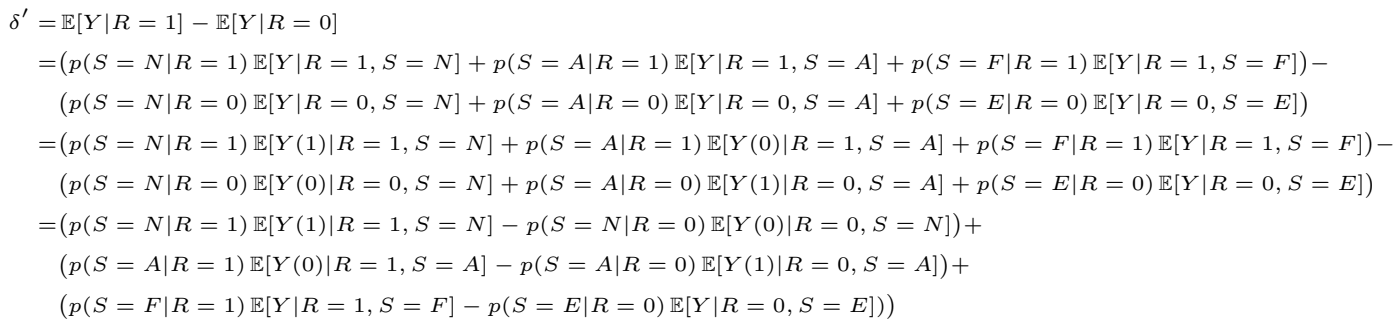

Under random sorting there are no escapees and no followers and $M$ (realized movement) is sufficient to characterize types:

$$
\begin{gathered}
=(p(M=0 \mid R=1) \mathbb{E}[Y(1) \mid R=1, M=0]-p(M=0 \mid R=0) \mathbb{E}[Y(0) \mid R=0, M=0])+ \\
(p(M=1 \mid R=1) \mathbb{E}[Y(0) \mid R=1, M=1]-p(M=1 \mid R=0) \mathbb{E}[Y(1) \mid R=0, M=1])
\end{gathered}
$$

For never-movers and always-movers, $Z$ is randomly assigned which implies that $R$ is randomly assigned for always- and never-movers:

$$
\begin{aligned}
= & (p(M=0 \mid R=1) \mathbb{E}[Y(1) \mid M=0]-p(M=0 \mid R=0) \mathbb{E}[Y(0) \mid M=0])+ \\
& (p(M=1 \mid R=1) \mathbb{E}[Y(0) \mid M=1]-p(M=1 \mid R=0) \mathbb{E}[Y(1) \mid M=1])
\end{aligned}
$$

Using the assumption of non-differential mover types:

$$
\begin{aligned}
= & (p(M=0 \mid R=1) \mathbb{E}[Y(1)]-p(M=0 \mid R=0) \mathbb{E}[Y(0)])+ \\
& (p(M=1 \mid R=1) \mathbb{E}[Y(0)]-p(M=1 \mid R=0) \mathbb{E}[Y(1)])
\end{aligned}
$$

Since $R$ and $M$ imply $Z$ for never- and always-movers: 


$$
\begin{aligned}
= & (p(Z=1 \mid R=1) \mathbb{E}[Y(1)]-p(Z=0 \mid R=0) \mathbb{E}[Y(0)])+ \\
& (p(Z=0 \mid R=1) \mathbb{E}[Y(0)]-p(Z=1 \mid R=0) \mathbb{E}[Y(1)])
\end{aligned}
$$

Re-arranging:

$$
\begin{aligned}
= & \mathbb{E}[Y(1)] p(Z=1 \mid R=1)+\mathbb{E}[Y(0)] p(Z=0 \mid R=1)- \\
& (\mathbb{E}[Y(1)] p(Z=1 \mid R=0)+\mathbb{E}[Y(0)] p(Z=0 \mid R=0)) \\
= & \mathbb{E}[Y(1)] p(Z=1 \mid R=1)+\mathbb{E}[Y(0)](1-p(Z=1 \mid R=1))- \\
& (\mathbb{E}[Y(1)] p(Z=1 \mid R=0)+\mathbb{E}[Y(0)](1-p(Z=1 \mid R=0))) \\
= & \mathbb{E}[Y(1)] p(Z=1 \mid R=1)+\mathbb{E}[Y(0)]-\mathbb{E}[Y(0)] p(Z=1 \mid R=1))- \\
& \mathbb{E}[Y(1)] p(Z=1 \mid R=0)-\mathbb{E}[Y(0)]+\mathbb{E}[Y(0)] p(Z=1 \mid R=0) \\
= & \mathbb{E}[Y(1)] p(Z=1 \mid R=1)-\mathbb{E}[Y(0)] p(Z=1 \mid R=1))- \\
& \mathbb{E}[Y(1)] p(Z=1 \mid R=0)+\mathbb{E}[Y(0)] p(Z=1 \mid R=0) \\
= & \mathbb{E}[Y(1)](p(Z=1 \mid R=1)-p(Z=1 \mid R=0))- \\
& \mathbb{E}[Y(0)](p(Z=1 \mid R=1)-p(Z=1 \mid R=0)) \\
= & (p(Z=1 \mid R=1)-p(Z=1 \mid R=0))(\mathbb{E}[Y(1)]-\mathbb{E}[Y(0)])
\end{aligned}
$$

\section{C.1.1 Random sorting with a stable population distribution}

Using the random exposure assumption, the incidental sorting assumption and the random sorting assumption as above:

$$
\begin{aligned}
\delta^{\prime}= & (p(Z=1 \mid R=1) \mathbb{E}[Y(1) \mid M=0]-p(Z=0 \mid R=0) \mathbb{E}[Y(0) \mid M=0])+ \\
& (p(Z=0 \mid R=1) \mathbb{E}[Y(0) \mid M=1]-p(Z=1 \mid R=0) \mathbb{E}[Y(1) \mid M=1])
\end{aligned}
$$

Since $p(Z=1)=p(R=1)$ :

$$
\begin{aligned}
& p(Z=1, R=1)=p(Z=0, R=0)=p(M=0) \\
& p(Z=1, R=0)=p(Z=0, R=1)=p(M=1)
\end{aligned}
$$

Therefore: 


$$
\begin{gathered}
=(p(M=0) \mathbb{E}[Y(1) \mid M=0]-p(M=0) \mathbb{E}[Y(0) \mid M=0])+ \\
(p(M=1) \mathbb{E}[Y(0) \mid M=1]-p(M=1) \mathbb{E}[Y(1) \mid M=1]) .
\end{gathered}
$$

Using the law of total expectation:

$$
\begin{aligned}
\mathbb{E}[Y(z)] & =\mathbb{E}[Y(z) \mid M=0] p(M=0)+\mathbb{E}[Y(z) \mid M=1] p(M=1) \\
& \Leftrightarrow \mathbb{E}[Y(z) \mid M=0]=1 / p(M=0)(\mathbb{E}[Y(z)]-\mathbb{E}[Y(z) \mid M=1] p(M=1))
\end{aligned}
$$

Substituting and re-arranging:

$$
\begin{aligned}
= & (\mathbb{E}[Y(1)]-\mathbb{E}[Y(1) \mid M=1] p(M=1)-\mathbb{E}[Y(0)]+\mathbb{E}[Y(0) \mid M=1] p(M=1))+ \\
& (p(M=1) \mathbb{E}[Y(0) \mid M=1]-p(M=1) \mathbb{E}[Y(1) \mid M=1]) \\
= & \mathbb{E}[Y(1)]-\mathbb{E}[Y(0)]-2 p(M=1)(\mathbb{E}[Y(1) \mid M=1]-\mathbb{E}[Y(0) \mid M=1])
\end{aligned}
$$

\section{C.2 Non-Random Sorting}

The derivation is the same as for random sorting except that we don't drop the term for escapees and followers.

$$
\begin{aligned}
\delta^{\prime}= & \mathbb{E}[Y \mid R=1]-\mathbb{E}[Y \mid R=0] \\
= & (\mathbb{E}[Y(1)]-\mathbb{E}[Y(0)])(p(Z=1 \mid R=1)-p(Z=1 \mid R=0))+ \\
& (p(S=F \mid R=1) \mathbb{E}[Y \mid R=1, S=F]-p(S=E \mid R=0) \mathbb{E}[Y \mid R=0, S=E])
\end{aligned}
$$

Since for all followers $R=1$ and for all escapees $R=0$ :

$$
\begin{aligned}
\delta^{\prime}= & (\mathbb{E}[Y(1)]-\mathbb{E}[Y(0)])(p(Z=1 \mid R=1)-p(Z=1 \mid R=0))+ \\
& (p(S=F) \mathbb{E}[Y \mid S=F]-p(S=E) \mathbb{E}[Y \mid S=E])
\end{aligned}
$$

